University of Nebraska - Lincoln

DigitalCommons@University of Nebraska - Lincoln

3-29-2007

\title{
Energetics of the dipole flip-flop motion in a ferroelectric polymer chain
}

Lei Cai

Florida International University, Icai@broward.org

Xuewen Wang

Florida International University, Miami, Florida

Yesim Darici

Florida International University

Jiandi Zhang

Florida International University, jiandiz@lsu.edu

Peter A. Dowben

University of Nebraska-Lincoln, pdowben@unl.edu

Follow this and additional works at: https://digitalcommons.unl.edu/physicsdowben

Part of the Physics Commons

Cai, Lei; Wang, Xuewen; Darici, Yesim; Zhang, Jiandi; and Dowben, Peter A., "Energetics of the dipole flipflop motion in a ferroelectric polymer chain" (2007). Peter Dowben Publications. 107.

https://digitalcommons.unl.edu/physicsdowben/107

This Article is brought to you for free and open access by the Research Papers in Physics and Astronomy at DigitalCommons@University of Nebraska - Lincoln. It has been accepted for inclusion in Peter Dowben Publications by an authorized administrator of DigitalCommons@University of Nebraska - Lincoln. 


\title{
Energetics of the dipole flip-flop motion in a ferroelectric polymer chain
}

\author{
Lei Cai, Xuewen Wang, Yesim Darici, and Jiandi Zhang \\ Department of Physics, Florida International University, Miami, Florida 33199
}

\section{P. A. Dowben}

Department of Physics and Astronomy, University of Nebraska-Lincoln, Lincoln, Nebraska 68588 and Center for Materials Research and Analysis, University of Nebrasca-Lincoln, Lincoln, Nebraska 68588

(Received 20 September 2006; accepted 19 January 2007; published online 29 March 2007)

\begin{abstract}
The authors report on a study of dipole flip-flop "local" transition in ferroelectric polyvinylidene fluoride $[\mathrm{P}(\mathrm{VDF})]$ chains, using total energy calculation based on the density functional theory. The calculated results indicate that a simple flipping of a single electric dipole moment is energetically allowed. Furthermore, such a flipping involves no change either in bond length, bond angle, or the orientation of the chain. The calculations also show that on a thin film of ordered chains, strong dipole interactions existing in $\mathrm{P}(\mathrm{VDF})$ could cause modulation of the dipole orientation thus forming superlattices on $\mathrm{P}(\mathrm{VDF})$ films. These results are in good agreement with recent scanning tunnel microscope experimental measurements. Furthermore, our calculations show that partial flipping may also exist and extend over a length of several monomers during the flip-flop transition. (C) 2007 American Institute of Physics. [DOI: 10.1063/1.2646731]
\end{abstract}

\section{INTRODUCTION}

Ferroelectric polymer materials with the reversibility of electric dipole moments provide a great opportunity to investigate dipole interactions and to explore technological applications. ${ }^{1}$ The crystalline polymer ferroelectric materials $^{2,3}$ are attractive because the complications such as compositional instabilities and lattice defects (point defects, steps, and grain boundaries) are greatly reduced. Among these materials, the ferroelectric polymer films of polyvinylidene fluoride $[\mathrm{P}(\mathrm{VDF})]$ and its copolymer films with vinylidene fluoride doped with trifluoroethylene [P(VDFTrFE)] are particularly interesting. The structural, electronic, and optical properties of $\mathrm{P}(\mathrm{VDF})$ or $\mathrm{P}(\mathrm{VDF}-\mathrm{TrFE})$ have been extensively studied experimentally ${ }^{4-17}$ and theoretically. ${ }^{18-21}$ The strong dipole moment existing in this material serves as an ideal playground for studying interesting phenomena such as field- ${ }^{3,4}$ and UV photoinduced ${ }^{22}$ molecular manipulations.

Recently, a transient reversal of local dipole moments (polarization) in the thin crystalline films of $\mathrm{P}(\mathrm{VDF}-\mathrm{TrFE})$ on graphite substrate has been demonstrated through the appearance of structural distortions induced by flipping the polarity of scanning tunnel microscope (STM) tip bias. ${ }^{4,5} \mathrm{~A}$ delicate $(4 \times 1)$ superstructure associated with the dipole orientation has also been observed, reflecting the intrinsic structural property of this crystalline copolymer film. On the other hand, there have been little theoretical investigations on these observed phenomena, especially the mechanism of the polarization manipulations. In this paper, we present results of an $a b$ initio total energy calculation on the structure changes associated with the dipole flip-flop transition in a $\mathrm{P}(\mathrm{VDF})$ chain and provide some detailed explanations to the previous experimental results obtained by STM. ${ }^{4,5}$

The basic structure of $\mathrm{P}(\mathrm{VDF})$ is a carbon chain with covalent bonding to $\mathrm{H}_{2}$ and $\mathrm{F}_{2}$ alternatively., ${ }^{2,18}$ Each monomer (unit) of $-\left(\mathrm{CH}_{2}-\mathrm{CF}_{2}\right)-$ has a large electric dipole mo- ment, pointing from electronegative fluorine to the electropositive hydrogen. At room temperature, $\mathrm{P}(\mathrm{VDF})$ is in the ferroelectric $\beta$ phase where dipole moments are mostly parallel to each other and roughly perpendicular to the chain axis $^{2}$ [see Fig. 1(a)], giving rise to the strong ferroelectric properties of $\mathrm{P}(\mathrm{VDF})$. On the other hand, the rotational free-
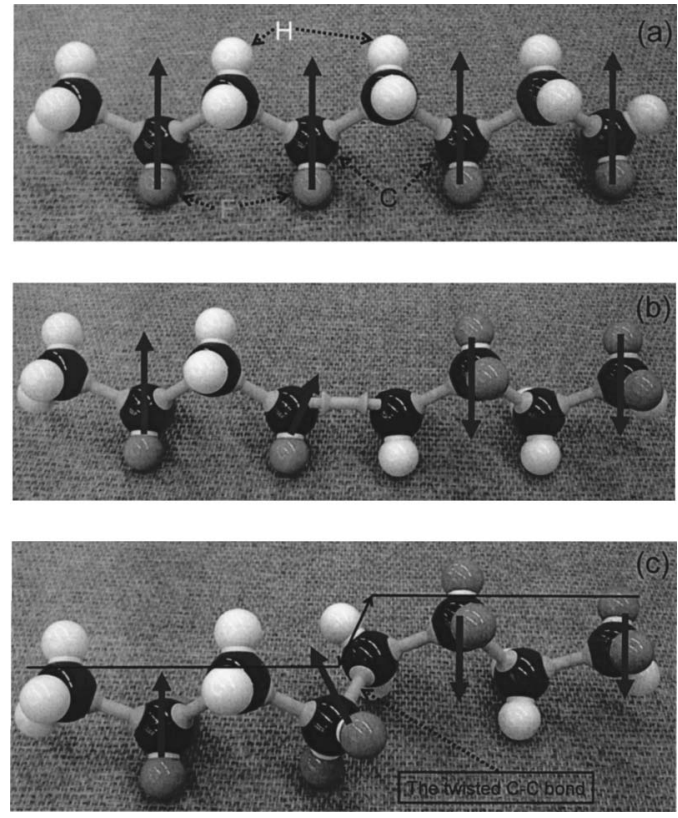

FIG. 1. (a) Side view of the ball structural model for an ideal P(VDF) chain in the all-trans configuration. Blue arrows indicate dipoles. (b) Side view of the model that the middle $\mathrm{C}-\mathrm{C}$ bond is cut and the half of the chain is flipped over $180^{\circ}$. Blue arrows indicate dipoles. (c) Side view of the ball structural model for a twisted $\mathrm{P}(\mathrm{VDF})$ chain when half of the chain was flipped over. Obviously, the rotated half-chain is parallel to the original chain. Blue arrows indicate dipoles. Solid black lines indicate that the half part of the chain is parallel to each other before and after the flipping. The twisted $\mathrm{C}-\mathrm{C}$ bond is also indicated in the figure. 
dom of individual dipole moment in the $\mathrm{P}(\mathrm{VDF})$ chain makes it energetically possible for the polarization manipulation in the system.

Experiments have shown that doping $\mathrm{P}(\mathrm{VDF})$ with TrFE can enhance the long-range structural ordering but does not change the basic polymer chain structure. ${ }^{2}$ Furthermore, both theoretical ${ }^{18}$ and experimental ${ }^{23}$ results have indicated that the replacement of $-\left(\mathrm{CH}_{2}-\mathrm{CF}_{2}\right)-$ with $-\left(\mathrm{CHF}-\mathrm{CF}_{2}\right)-$ only changes slightly the band structure and does not substantially affect the band gap between the highest occupied and the lowest unoccupied molecular orbitals. Therefore, we chose $\mathrm{P}(\mathrm{VDF})$ instead of $\mathrm{P}(\mathrm{VDF}-\mathrm{TrFE})$ as the model polymer in our study. This avoids complexity in the model calculations due to the involvement of TrFE and enhances clear insights of energetics of the "local" dipole flip-flop motion.

\section{THEORETICAL APPROACH AND MODEL STRUCTURE}

Standard density functional theory (DFT) for quantum chemistry ${ }^{24}$ is used to study the structures and structural distortions. The local density approximation is used for the exchange-correlation potential. ${ }^{25}$ Electronic structures as well as total energies are obtained using the first-principles calculation. The work reported here emphasizes the structure and total energy [a strength of DFT in modeling P(VDFTrFE) (Ref. 26)], rather than the electronic excitation (where DFT does not do very well). The $\mathrm{P}(\mathrm{VDF})$ polymer is modeled by a chain consisting of four or five $-\left(\mathrm{CH}_{2}-\mathrm{CF}_{2}\right)$ monomers, as has been successfully done elsewhere with semiempirical model calculations of $\mathrm{P}(\mathrm{VDF}-\mathrm{TrFE}){ }^{23,27}$ The carbon atoms at the two ends of the chain are capped with hydrogen atoms. This is a typical technique used in other calculations ${ }^{23,26-28}$ in order to correct the finite size effect and to compensate the loss of covalent bonding. A linear combination of atomic orbitals centered on each atom is used to expand the electronic wave functions. Three $s$-type and two $p$-type orbitals are used for both $\mathrm{F}$ and $\mathrm{C}$ atoms, while two $s$-type orbitals are used for $\mathrm{H}$ atoms. A series of Gaussian orbitals is used to represent the atomic orbitals. ${ }^{29,30}$ This setup gives rise to a total of 164 atomic orbitals and 696 primitive Gaussian orbitals for the calculation of a chain with four $-\left(\mathrm{CH}_{2}-\mathrm{CF}_{2}\right)-$ monomers.

The ideal structure of the polymer chain is assumed to be the $\beta$ phase structure with the dipole moments parallel to each other, as shown in Fig. 1(a). All bond lengths and bond angles are determined by total energy calculations. We fully relax the structure of the four-monomer chain through energy minimization. The calculated dipole moment per monomer (1.6 D) as well as structural parameters are all found to be in good agreement with other studies. ${ }^{20,31-33}$ All energy values reported in this paper are measured with respect to the total energy of the ideal structure.

\section{CALCULATED RESULTS AND DISCUSSIONS}

To study local dipole flip-flop transitions of the $\mathrm{P}(\mathrm{VDF})$ polymer chain, we first examine a twisted chain where half of the chain is flipped $180^{\circ}$. The simplest model is to sever the middle $\mathrm{C}-\mathrm{C}$ bond and flip half of the chain over [see Fig.

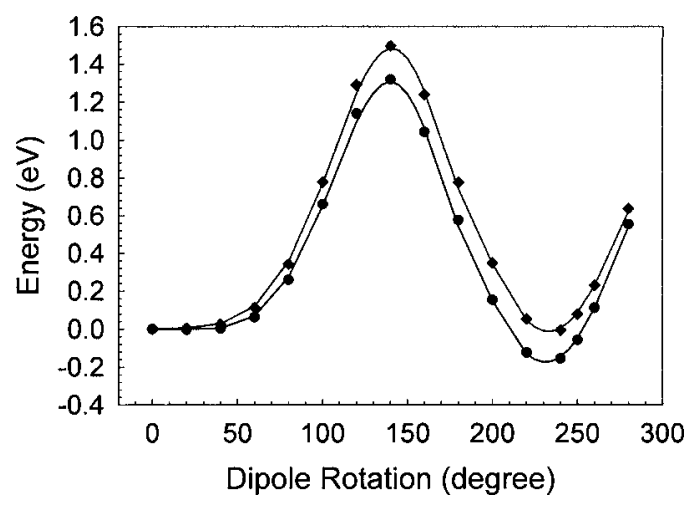

FIG. 2. The calculated total energy of a $\mathrm{P}(\mathrm{VDF})$ chain as a function of the dipole rotation around the chain axis where $0^{\circ}$ is for the ideal structure and $180^{\circ}$ for the flipping over structure. The black solid circles indicate the calculated energy and the red solid diamonds indicate the energy including the effect of the interactions. All energy values are measured with respect to the total energy of the ideal structure. The solid line guides the eyes.

1(b)]. The length of the middle $\mathrm{C}-\mathrm{C}$ bond is adjusted to minimize the total energy. The calculations show that the total energy of this flipped structure is higher than that of the ideal structure by $2.5 \mathrm{eV}$. This is to be expected because the above flipping model changes the bond angles at the middle of the chain, as shown in Fig. 1(b), and it is known that the $\mathrm{C}-\mathrm{C}$ tetrahedral covalent bond angle is fairly rigid. Notice also that the $2.5 \mathrm{eV}$ is very likely the minimum energy required to flip half the chain. Some intermediate structures that may occur during the flipping transition could require even higher energies due to large changes in the $\mathrm{C}-\mathrm{C}$ bond angles and lengths. The results of this simple model have prompted us to search for a flipping model that requires no change in bond length and bond angle.

The position of any carbon atom in the $\mathrm{P}(\mathrm{VDF})$ chain is determined by three degrees of freedom. If the $\mathrm{C}-\mathrm{C}$ bond lengths and angles are fixed, the only remaining degree of freedom is the dihedral angle. By changing the dihedral angles of carbon chain, half of the polymer chain can be twisted continuously until it is flipped over [as shown in Fig. $1(\mathrm{c})]$. For a covalent bond system such as P(VDF), the dominant electronic energies are the $s p^{3}$ hybridization energy, which critically depends on the tetrahedral bond angle, and the nearest-neighbor $\sigma$ bonding energy, which depends on the bond length. A local flipping transition model that leaves all bond angles and bond lengths unperturbed will cost the least amount of electronic energy. For convenience, the amount of twisting of the chain is measured by a rotational angle $\theta$. It is the rotational angle of the dipole moment about the chain axis. Notice from Fig. 1(c), in order to preserve $\mathrm{C}-\mathrm{C}$ bond lengths and angles, that the position of the flipped half-chain must be shifted relative to the unflipped part.

The total energies of the whole chain were calculated as a function of rotational angle $\theta$ and the results are shown in Fig. 2. From these calculations, we find that the energy of the flipped structure at $\theta=180^{\circ}$ is higher than that of the ideal structure by $0.58 \mathrm{eV}$. This means that the flipped structure at $180^{\circ}$ is not stable and the flipped dipoles could not be maintained without external forces. The energy barrier for the flipping transition, occurring around $140^{\circ}$, is even higher at 
about $1.3 \mathrm{eV}$ above the energy of the ideal structure. Rotational angles larger than $180^{\circ}$ were also calculated, and the lowest energy structure was found at $235^{\circ}$ (see Fig. 2, energy versus $\theta$ ), where the energy is lower than the ideal structure by $0.17 \mathrm{eV}$.

The energy of the structure with a locally flipped electric dipole at $235^{\circ}$ is lower than that of the ideal structure. This implies that the flipped structure at $235^{\circ}$ is a more stable structure. It should be mentioned that the above results are obtained for a single, isolated chain of $\mathrm{P}(\mathrm{VDF})$. With dihedral angle being the only degree of freedom, the $\mathrm{P}(\mathrm{VDF})$ chain is a truly one-dimensional system, provided that interchain hybridization and steric hindrance effects can be neglected. It is known that a one-dimensional system has no long-range order. So for a thin film of $\mathrm{P}(\mathrm{VDF})$ the interchain interaction as well as the interaction with the substrate should be taken into account to obtain a stable ordered structure. These interactions can be described approximately by an effective electric field $\boldsymbol{E}$. This effective electric field will contribute an additional term of $|\boldsymbol{E}| \cdot|\boldsymbol{P}| \cdot(1-\cos (\theta))$ to the total energy, with $\boldsymbol{P}$ being the dipole moment of the flipped half-chain. This additional term will lift the total energy of the flipped structure at $\theta=235^{\circ}$ such that the energy is no longer less than that of the ideal structure $\left(\theta=0^{\circ}\right)$. This scenario is indicated in Fig. 2 (see the red solid diamonds as compared with the black solid circles). Assuming that the total energy of the structure with a locally flipped electric dipole at $\theta=235^{\circ}$ equals that of the ideal structure, we can estimate that the effective field should be around $0.3 \mathrm{~V} / \AA$.

We have also evaluated the length change of the entire chain due to flipping. We find that although bond lengths and bond angles are not changed, the total length of the chain is shortened by $0.64 \AA$ due to one twisted $\mathrm{C}-\mathrm{C}$ bond [indicated in Fig. 1(c)]. This will cause some stress along the chain. In addition, we find that the position of the flipped half-chain is shifted perpendicularly to the original chain by $1.43 \AA$.

There are two important features in this flipping model. Firstly, as being discussed earlier, it requires no change either in bond length or bond angle of all covalent $\mathrm{C}-\mathrm{C}, \mathrm{C}-\mathrm{H}$, and $\mathrm{C}-\mathrm{F}$ bonds during the entire transition; thus the energy cost is likely the lowest in comparison with other possible flipping models. Secondly, as shown in Fig. 1(c), the flipped half-chain remains perfectly parallel to the surface as well as to the original chain direction. In fact, the half-chain remains parallel to the original chain direction at any angles of rotation. This feature allows a layer of parallel polymer chains to rotate and flip simultaneously. These two key features make it possible to achieve smooth and reversible flip-flop transitions in an ordered ferroelectric polymer film.

The recent STM experiments ${ }^{4,5}$ have observed that the thin films of $\mathrm{P}(\mathrm{VDF}-\mathrm{TrFE})$ could form well-ordered crystalline or parallel chain structures on graphite substrates. With a relatively larger tip bias thus a strong electric field, the STM images have indicated that $\mathrm{P}(\mathrm{VDF}-\mathrm{TrFE})$ thin film surface exhibits ferroelectric $\beta$-phase-like structure (i.e., with the dipoles largely aligned). By switching the polarity of STM tip bias, the dipole moments of the copolymer chains can be manipulated by the STM tip, accompanied by a change of structure similar to the theoretical model described above. It has been observed ${ }^{4,5}$ that the flipped part of the chain remains parallel to the unflipped part in STM images but with an apparent lattice shift in the direction perpendicular to the polymer chains during the flipping manipulation. The experimental displacement of the chains is observed to be $\sim 1.65 \AA$, slightly larger than the theoretical value of $1.43 \AA$. Within the accuracy of STM measurements, no change in the interchain or intrachain spacing is observed with the STM tip induced dipole reorientation. ${ }^{4,5}$ These results are in good agreement with our theoretical calculations showing that the dipole flipping involves no change in bond length or bond angle.

Another interesting feature in Fig. 2 is that the total energy is essentially constant until the rotational angle reaches about $40^{\circ}$. In fact, there is a very shallow energy minimum at small angles. The origin of this energy minimum is due to the competition between the electronic energy and the repulsive dipole-dipole interaction. The electronic energy favors the ideal structure, while the dipole repulsion forces the dipoles to misalign. For a single polymer chain, the dipole repulsion wins a little. In the thin film of $\mathrm{P}(\mathrm{VDF})$, as being discussed above, additional interactions will contribute an additional energy term of $|\boldsymbol{E}| \cdot|\boldsymbol{P}| \cdot(1-\cos (\theta))$. This latter term pushes the energy minimum towards smaller $\theta$ values, or even eliminates it and stabilizes the structure of the thin film at the ideal structure $\left(\theta=0^{\circ}\right)$.

One might speculate that if the off-normal orientation energy minimum is not eliminated completely, then the adjacent dipoles will not be perfectly aligned. Such an offnormal orientation energy minimum should lead to a canted phase or even a superstructure of the dipole orientation along the chain. We believe that this scenario may have already been verified by the experimental results. In a recent STM study of $\mathrm{P}(\mathrm{VDF}-\mathrm{TrFE})$ film deposited on graphite, a delicate $(4 \times 1)$ superlattice is indeed imaged under very small tip bias $^{5}$ at room temperature. Using low tip bias minimizes the effect of the tip field on the intrinsic structure. This $(4 \times 1)$ superlattice can be described reasonably well as a periodic canting of $-\left(\mathrm{CH}_{2}-\mathrm{CF}_{2}\right)$ - dipole resulted from a small $\theta$ modulation along the polymer chain. Better electric dipole alignment, demonstrated by a variety of experiments undertaken at low temperatures, may be the origin of, or the consequence of, lattice stiffening transition of crystalline $\mathrm{P}(\mathrm{VDF}-\mathrm{TrFE})$ in the temperature region of $160 \mathrm{~K}^{34,35}$

With the flipping of the half-chain, even though all bond lengths and bond angles are unaltered, the change in the $\mathrm{C}-\mathrm{C}$ bond orientation causes the full chain length to shrink by $0.64 \AA$, which is almost $\frac{1}{4}$ of the intrachain spacing of $2.6 \AA^{2}{ }^{2}$ This contraction of the chain is not evident in STM measurements. ${ }^{4}$ In order to understand this apparent discrepancy, we need to realize that the current calculations are based on the model of a single, isolated, and freestanding chain where the half-chain flipping involves only one twisted $\mathrm{C}-\mathrm{C}$ bond. This may not be exactly the case in STM experiments. In the STM polarization manipulation through tip field, only a finite section of polymer chain underneath the STM tip is reoriented, while the rest of polymer chain remains unperturbed. The length of the rotated section depends on the strength of the external field determined by the size, 

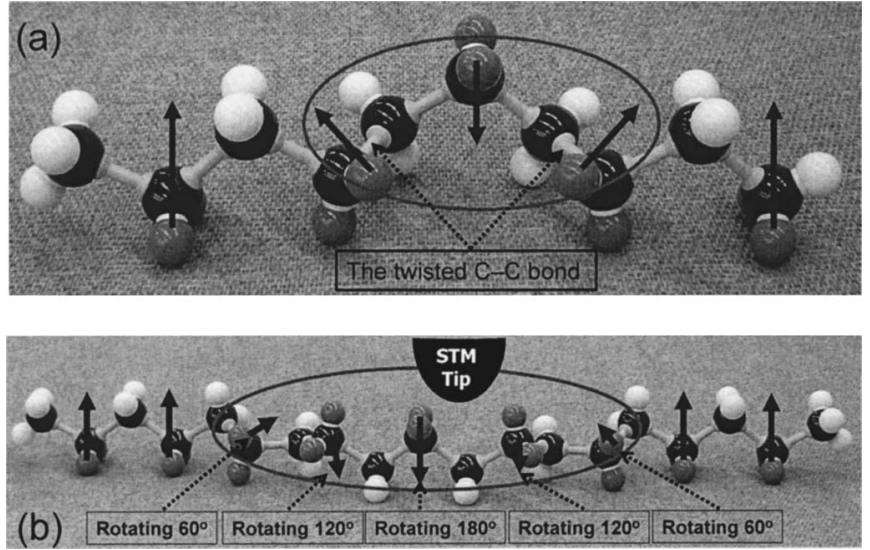

FIG. 3. Ball structural model for a (a) polarization soliton appearing in the center of the chain shown in the red ellipse with the indication of two twisted $\mathrm{C}-\mathrm{C}$ bonds; (b) "dressed" soliton involving five $\mathrm{C}-\mathrm{C}$ bonds, with $60^{\circ}$ difference between nearest neighbors (shown in the red ellipse). Blue arrows indicate dipoles.

shape, and bias of the STM tip. Therefore, in such a sectional flipping scenario, the achievement of reorienting $\mathrm{P}(\mathrm{VDF})$ section should require at least two $\mathrm{C}-\mathrm{C}$ bonds to be twisted [indicated in Fig. 3(a)]. The strain created could be distributed over a large section of the $\mathrm{P}(\mathrm{VDF})$ chain, or the strain in the chain could be stabilized by the interactions with the substrate, thus exhibiting no large contraction of the chain.

Considering the realistic scenarios for the STM tip induced sectional dipole flip-flop transition of $\mathrm{P}(\mathrm{VDF})$ chains, we propose two electric dipole flipping models, with different numbers of dipoles involved in the (re)polarization process. One model is the simple polarization soliton model consisting of one completely flipped dipole moment plus two tilted ones [see Fig. 3(a)]. The other is the so called dressed polarization soliton model in which the flipped section contains more dipoles that are flipped or partially flipped [see Fig. 3(b)]. Based on our calculations (see Fig. 2), there are negligible energy costs to have canted dipoles with a small rotational angle $(\theta)$. Thus a dressed polarization soliton should be more favorable in energy and strain considerations when a section of chain is reoriented.

In order to obtain quantitatively insight mechanisms for dipole reorientation, we first carried out the total energy calculation for the simplest bare polarization soliton model. To do so, we use a longer chain containing five $-\left(\mathrm{CH}_{2}-\mathrm{CF}_{2}\right)-$ monomers. The calculation involves 204 atomic orbitals but a smaller Gaussian basis with 744 primitive Gaussian orbitals. Again, all bond lengths and bond angles are conserved during the entire dipole reorientation transition. Our calculations show that the energy cost for forming a single polarization soliton is $1.06 \mathrm{eV}$. It is worthwhile to notice that such a polarization soliton have two twisted $\mathrm{C}-\mathrm{C}$ bonds, thus the energy cost $(\sim 1.06 \mathrm{eV})$ should be close to twice the energy cost of the half-chain flipping $(0.58 \mathrm{eV})$, as being the case here. Since there are two twisted bonds in the model, the formation of such a soliton causes an even larger contraction of the chain length by $1.28 \AA$. The change of dipole moment caused by the creation of a polarization soliton is calculated to be about $4 \mathrm{D}$. Without considering the effective electric field mentioned earlier, we estimate that an electric field of $1.3 \mathrm{~V} / \AA$ is needed to create one such polarization soliton. This electric field strength is much stronger than the upper limit of the electric field, $0.3 \mathrm{~V} / \AA$, estimated from experiments. ${ }^{4,5}$ Overall, the larger reduction of the chain length and the stronger electric field indicate that such a "naked" polarization soliton model is not really suitable to explain the STM tip induced dipole flipping in $\mathrm{P}(\mathrm{VDF})$ chains.

To form a simple but "dressed" soliton, we let adjacent dipoles on both sides of the above polarization soliton to rotate, as shown schematically in Fig. 3(b). Such a dressed soliton involves rotation of five dipoles, with $60^{\circ}$ between the nearest neighbors, such that the electric dipole orientation at the middle of the chain is aligned opposite to the original dipole orientation. Our calculations show that the energy cost is only $0.42 \mathrm{eV}$ and the required electric field strength is only $0.5 \mathrm{~V} / \AA$ for the creation of such a dressed soliton. This estimate of the electric field is much closer to the experimental estimate of $0.3 \mathrm{~V} / \AA$. Such a dressed soliton model not only reduces the energy cost but also reduces the strain along the chain. Instead of shortening of $1.28 \AA$, the polymer chain is only contracted by $0.28 \AA$. It can be foreseen that an even longer dressed soliton involving more rotated dipole moments will have even smaller energy cost and length contraction. Therefore, the dressed soliton model is likely the true scenario for the spontaneous dipole flip-flop transition resulted from manipulation by the STM tip.

Both the dressed and undressed solitons appear to cost far less energy than the $3.66 \mathrm{eV}$ photon energy used to "locally" flip an electric dipole in crystalline P(VDF-TrFE), ${ }^{22}$ and therefore are not energetically forbidden in those experiments. The mechanisms for the photoexcitation leading to a locally flipped electric dipole in crystalline $\mathrm{P}(\mathrm{VDF}-\mathrm{TrFE})$ (Ref. 22) may not, however, be easily identified as those initiated by the STM tip. Nevertheless, either the dressed or undressed solitons are likely transient states resulting from photoexcitation, leading to a locally flipped electric dipole in crystalline $\mathrm{P}(\mathrm{VDF}-\mathrm{TrFE}){ }^{22}$

\section{SUMMARY}

We studied the energetics of the dipole flip-flop transition in ferroelectric $\mathrm{P}(\mathrm{VDF})$ polymer chain using several possible structural models. We have demonstrated that the rotation of local dipole moments is an energetically favorable mechanism and that the rotation can occur without any significant change in bond length or bonding angle. Hence, the dipole flip-flop transition could be initiated by small local electric fields. Due to the competition between electronic energy and dipole-dipole repulsion as well as the adlayersubstrate interactions, the $\mathrm{P}(\mathrm{VDF})$ chains may adopt a canted phase with a small amount of $\mathrm{C}-\mathrm{C}$ bond twisting, particularly at finite temperatures, ${ }^{34}$ which give rise to a superlattice structure, as observed on the thin film surface. Our calculations also indicate that the dressed polarization soliton formation is the energetically favorable scenario for the actual flipping of the dipoles in the thin film caused by the STM tip or by photoexcitation leading to a "locally" flipped electric dipole in crystalline $\mathrm{P}(\mathrm{VDF}-\mathrm{TrFE})$. Furthermore, it is found 
that the dressed polarization soliton could be composed of as fewer as five dipoles, which means that the dipole moment reversal could be localized on a polymer thin film without affecting large areas of the film. Thus the feasibility of the local polarization provides the theoretical evidence for the application of $\mathrm{P}(\mathrm{VDF})$ or $\mathrm{P}(\mathrm{VDF}-\mathrm{TrFE})$ thin film in highdensity molecular memory devices.

\section{ACKNOWLEDGMENT}

Support by the National Science Foundation through Grant Nos. CHE 0415421 and DMR 0346826 is gratefully acknowledged.

${ }^{1}$ H. S. Nalwa, Ferroelectric Polymers: Chemistry, Physics, and Applications (Dekker, New York, 1995).

${ }^{2}$ L. M. Blinov, V. M. Fridkin, S. P. Palto, A. V. Bune, P. A. Dowben, and S. Ducharme, Usp. Fiz. Nauk 170, 247 (2000); [Phys. Usp. 43, 243 (2000)].

${ }^{3}$ S. Ducharme, S. P. Palto, and V. M. Fridkin, Ferroelectric and Dielectric Films, Handbook of Surfaces and Interfaces of Materials Vol. 3 (Academic Press, Los Angeles, 2002), pp. 546-592.

${ }^{4}$ H. Qu, T. Garcia, W. Yao, J. Zhang, S. Ducharme, P. A. Dowben, A. V. Sorokin, and V. M. Fridkin, Appl. Phys. Lett. 82, 4322 (2003).

${ }^{5}$ L. Cai, H. Qu, C. Lu, S. Ducharme, P. A. Dowben, and J. Zhang, Phys. Rev. B 70, 155411 (2004).

${ }^{6}$ K. Matsushige, H. Yamada, H. Tanaka, T. Horiuchi, and X. Q. Chen, Nanotechnology 9, 208 (1998).

${ }^{7}$ J. Choi, C. N. Borca, P. A. Dowben et al., Phys. Rev. B 61, 5760 (2000).

${ }^{8}$ X. Q. Chen, H. Yamada, Y. Terai, T. Horiuchi, K. Matsysguge, and P. S. Weiss, Thin Solid Films 353, 259 (1999).

${ }^{9}$ T. Fukuma, K. Kobayashi, T. Horiuchi, H. Yamada, and K. Matsushige, Jpn. J. Appl. Phys., Part 1 39, 3830 (2000).

${ }^{10}$ K. Kimura, K. Kobayashi, H. Yamada, T. Horiuchi, K. Ishida, and K. Matsushige, Appl. Phys. Lett. 82, 4050 (2003).

${ }^{11}$ K. Kobayashi, H. Masuda, H. Yamada, and K. Matsushige, Eur. Polym. J. 40, 987 (2004).

${ }^{12}$ J. Choi, S.-J. Tang, P. T. Sprunger, P. A. Dowben, V. M. Fridkin, A. V. Sorokin, S. P. Palto, N. Petukhova, and S. G. Yudin, J. Phys.: Condens.
Matter 12, 4735 (2000).

${ }^{13}$ C. N. Borca, S. Adenwalla, J. Choi et al., Phys. Rev. Lett. 83, 4562 (1999).

${ }^{14}$ J. Choi, P. A. Dowben, S. Pebley, A. V. Bune, S. Ducharme, V. M. Fridkin, S. P. Palto, and N. Petukhova, Phys. Rev. Lett. 80, 1328 (1998).

${ }^{15}$ O. A. Aktsipetrov, L. M. Blinov, V. M. Fridkin, T. V. Misuryaev, T. V. Murzina, S. P. Palto, and S. G. Yudin, Surf. Sci. 454-456, 1016 (2000).

${ }^{16}$ Q. M. Zhang, V. Bharti, and X. Zhao, Science 280, 2101 (1998).

${ }^{17}$ F. Xia, H. Xu, F. Fang, B. Razivi, Z. Y. Cheng, Y. Lu, B. Xu, and Q. M. Zhang, Appl. Phys. Lett. 78, 1122 (2001).

${ }^{18}$ C.-g. Duan, W. N. Mei, J. R. Hardy, S. Ducharme, J. Choi, and P. A. Dowben, Europhys. Lett. 61, 81 (2003).

${ }^{19}$ H. von Seggern and G. F. Leal Ferreira, Appl. Phys. Lett. 83, 3353 (2003).

${ }^{20}$ C.-g. Duan, W. N. Mei, W.-g. Yin, J. Liu, J. R. Hardy, S. Ducharme, and P. A. Dowben, Phys. Rev. B 69, 235106 (2004).

${ }^{21}$ H. Su, A. Strachan, and W. A. Goddard III, Phys. Rev. B 70, 064101 (2004).

${ }^{22}$ L. G. Rosa, P. A. Jacobson, and P. A. Dowben, J. Phys. Chem. B 110, 7944 (2006)

${ }^{23}$ J. Choi, E. Morikawa, S. Ducharme, and P. A. Dowben, Mater. Lett. 59, 3599 (2005).

${ }^{24}$ R. O. Jones and O. Gunnarsson, Rev. Mod. Phys. 61, 689 (1989).

${ }^{25}$ D. M. Ceperley and B. J. Alder, Phys. Rev. Lett. 45, 566 (1980).

${ }^{26}$ L. G. Rosa, J. Xiao, Ya. B. Losovyj, Y. Gao, I. N. Yakovkin, X. C. Zeng, and P. A. Dowben, J. Am. Chem. Soc. 127, 17261 (2005).

${ }^{27}$ J. Xiao, L. G. Rosa, M. Poulsen, D.-Q. Feng, S. Reddy, J. M. Takacs, L. Cai, J. Zhang, S. Ducharme, and P. A. Dowben, J. Phys.: Condens. Matter 18, L155 (2006).

${ }^{28}$ K. N. Ow and X. W. Wang, Surf. Sci. 337, 109 (1995).

${ }^{29}$ R. Ditchfield, W. J. Hehre, and J. A. Pople, J. Chem. Phys. 54, 724 (1971).

${ }^{30}$ P. C. Hariharan and J. A. Pople, Theor. Chim. Acta 28, 213 (1973).

${ }^{31}$ C. K. Purvis and P. L. Taylor, Phys. Rev. B 26, 4547 (1982).

${ }^{32}$ C. K. Purvis and P. L. Taylor, J. Appl. Phys. 54, 1021 (1983).

${ }^{33}$ L. G. Rosa, I. N. Yakovkin, and P. A. Dowben, J. Phys. Chem. B 109, 14189 (2005)

${ }^{34}$ L. G. Rosa, Ya. B. Losovyj, J. Choi, and P. A. Dowben, J. Phys. Chem. B 109, 7817 (2005)

${ }^{35}$ C. N. Borca, S. Adenwalla, J. Choi et al., Phys. Rev. Lett. 83, 4562 (1999). 